\title{
HyperScatter - Modellierungs- und Zoomtechniken für Punktdiagramme
}

\author{
Jens Gerken, Mischa Demarmels, Stefan Dierdorf und Harald Reiterer \\ Arbeitsgruppe Mensch-Computer Interaktion, Universität Konstanz
}

\section{Zusammenfassung}

Der Einsatz von Werkzeugen aus dem Bereich der Informationsvisualisierung im privaten Alltag, beispielsweise beim Stöbern in digitalen Bibliotheken wie einer Filmdatenbank, stellt Entwickler vor neue Herausforderungen bezüglich des Interaktionsdesigns - muss dieses doch den deutlich höheren Anforderungen der zumeist unerfahrenen Nutzer hinsichtlich der Gebrauchstauglichkeit genügen. Ein möglicher Lösungsansatz könnte hierbei in dem kombinierten Einsatz möglichst einfacher Werkzeuge, die auf einem einheitlichen Interaktionsdesign basieren, liegen. Dieser Beitrag führt die Arbeit aus (Reiterer et al. 2005) fort und zeigt am Beispiel eines Punktdiagramms, dem HyperScatter auf, wie die dort vorgestellte Modellierung des Informationsraums in Kombination mit Zoomtechniken auf andere Werkzeuge übertragbar ist und hierdurch das Ziel eines einheitlichen Interaktionsdesigns erreicht wird. Weiterhin wurde der HyperScatter in einer neuartigen zweiwöchigen Längsschnitt-Evaluationsstudie auf seine Gebrauchstauglichkeit hin überprüft - sowie auf seine Fähigkeit, als alleinstehendes Werkzeug den Benutzer in seiner Informationssuche zu unterstützen.

\section{$1 \quad$ Einleitung}

Die Informationsvisualisierung steht vor einer neuen Herausforderung. Waren bislang zumeist geschulte Analysten auf die spezialisierten visuellen Analysewerkzeuge angewiesen, so könnten heute auch die privaten Endanwender enorm von deren mächtigen Fähigkeiten große Datenräume visuell darzustellen, profitieren. Denn die digitalen privaten Informationsräume sind einem enormen Wachstum unterworfen, seien es e-Mails, persönliche Dateien oder auch öffentlich zugängliche Informationsräume wie digitale Bibliotheken (z.B. eine Filmdatenbank). Um sich hier zu recht zu finden, reichen klassische Werkzeuge wie Desktopsuchen mit listenbasierten Trefferdarstellungen kaum noch aus; zu heterogen, umfangreich und komplex sind mittlerweile nicht nur die Daten selbst, sondern auch deren Metadaten. Die Informationsvisualisierung könnte durch ihre langjährige Erfahrung entscheidend dazu beitragen, diese Situation zu verbessern. Dabei muss sie allerdings den Ansprüchen der 
Privatanwender gerecht werden und verstärkt auf einfache Benutzbarkeit und Erlernbarkeit Wert legen. Ein Lösungsansatz könnte in der Kombination möglichst einfacher Werkzeuge liegen, die im Zusammenspiel dennoch eine hohe Mächtigkeit in ihrer Analysefähigkeit bieten. Dieser Ansatz wird im Rahmen des Projekts MedioVis verfolgt, in welchem ein gleichnamiges visuelles Suchsystem entwickelt wurde, welches eine Filmdatenbank über verschiedene Werkzeuge dem Benutzer zugänglich macht (Gruen et al. 2005). Ein Werkzeug in MedioVis stellt die HyperGrid dar, welche eine tabellenbasierte Darstellung um semantisches Zooming und die Möglichkeit der Einbettung von beliebigen Webservices erweitert. Hierdurch wird neben einem zielgerichteten Suchprozess auch ein interessengeleitetes Stöbern im Informationsraum unterstützt. Die HyperGrid greift hierbei auf eine semantische Modellierung des Informationsraums zurück, der Attributraummodellierung, wie in (Reiterer et al. 2005) erstmals vorgestellt. Sollen mehrere Werkzeuge wie in MedioVis miteinander kombiniert werden, ist es von entscheidender Bedeutung, dass diese ein einheitliches Interaktionskonzept aufweisen. Hierdurch kann vermieden werden, dass der Benutzer ständig umzudenken hat und letztlich die Einfachheit der einzelnen Werkzeuge durch die Komplexität des Gesamtsystems verloren geht. Eine einheitliche Attributraumodellierung in Kombination mit Zoomtechniken könnte eine entscheidende Voraussetzung für ein derartiges homogenes Interaktionskonzept sein. In diesem Beitrag soll daher die Übertragbarkeit der in Reiterer et al. vorgestellten Attributraummodellierung und Zoomtechniken auf andere Darstellungsformen anhand eines Punktdiagramms, dem HyperScatter, demonstriert werden. In Kapitel 2 werden zunächst Punktdiagramme im Allgemeinen vorgestellt, um einen Überblick über den Stand der Forschung zu geben. In Kapitel 3 wird daraufhin der HyperScatter im Detail vorgestellt, wobei insbesondere der Frage der nahtlosen Integration in ein Gesamtsystem Rechnung getragen werden soll. Im Kapitel 4 wird eine Evaluationsstudie vorgestellt, in welcher der HyperScatter der HyperGrid gegenübergestellt wurde. Überprüft wurde darüberhinaus auch, inwieweit das Konzept alleine tragfähig ist oder dem Benutzer eher in Ergänzung angeboten werden sollte.

\section{2 Der HyperScatter}

Papierbasierte Punktdiagramme werden seit dem frühen 19. Jahrhundert verwendet, um abstrakte Daten abzubilden. Diese werden anhand zweier Dimensionen in einem Koordinatensystem verortet, wodurch sowohl ein Überblick über den Datenraum möglich wird als auch das schnelle Erkennen von Zusammenhängen und Trends. (Meyer \& Shinar 1991) konnten hierbei aufzeigen, dass dies auch ungeübten Nutzern problemlos möglich ist, also keine Vorkenntnisse in der Datenanalyse erforderlich sind. Zurückzuführen ist diese Erkenntnis möglicherweise auf die generelle Vertrautheit mit der Darstellung, beispielsweise aus der Mathematik. Dementsprechend werden Punktdiagramme auch heute noch vielfach eingesetzt um Daten grafisch aufzubereiten. In der Disziplin der Informationsvisualisierung wurde das Punktdiagramm von (Ahlberg \& Shneiderman 1994) erstmals um Interaktionselemente bereichert. Die prinzipielle Abbildung von Datenpunkten in einem zweidimensionalen Koordinatensystem wurde hierbei um einen entscheidenden Aspekt erweitert: der Möglichkeit, interaktiv die Skalierung zu ändern und somit dichte Anhäufungen von Datenpunkten auflö- 
sen zu können. Ahlberg \& Shneiderman nannten das entstandene Werkzeug fortan „Starfield Display“, welches sie zudem noch um dynamische Filtermöglichkeiten, den „dynamic queries" erweiterten. Aufgrund der effizienten Platznutzung skaliert das Konzept besonders gut auf unterschiedliche Bildschirmgrößen. Erfolgreiche Beispiele für kleine Displays sind der PalmMovieFinder (Dunlop \& Davidson 2000) oder das Liquid Browsing Konzept von (Waldeck \& Balfanz 2004). Letzteres beinhaltet auch ein alternatives Konzept um dichte Anhäufungen von Datenpunkte aufzulösen. Vernachlässigt wurden in den meisten Ansätzen jedoch die Präsentation der Datenpunkte selbst und die Interaktion mit diesen. Hier offenbart das ursprüngliche Konzept des Filmfinders eklatante Schwächen, kann es doch nur unzureichend den heutigen Herausforderungen, wie zum Beispiel der hohen Dimensionalität und Heterogenität der zugrundeliegenden Metadaten, gerecht werden. Ebenso wenig ist es möglich, tiefer in den Datenraum einzutauchen und beispielsweise mehr über einen bestimmten Film oder auch Schauspieler zu erfahren, entsprechend dem eigenen Interesse. Dieser Umstand definiert zugleich auch die Anforderung an das HyperScatter Konzept. Zum einen soll das Filmfinder Konzept zeitgemäß umgesetzt werden, was sich insbesondere in der Zoominteraktion niederschlagen soll. Weiterhin soll es dem Benutzer möglich sein, nicht nur in den Datenraum selbst sondern auch in einzelne Datenpunkte einzutauchen und hier interessenspezifisch auf Informationen zugreifen zu können. Neben den analytischen Fähigkeiten des Punktdiagramms soll so auch eine interessengeleitete Exploration des Informationsraums unterstützt werden.

\subsection{Zoomtechniken in Punktdiagrammen}

In dem von uns im Projekt MedioVis angestrebten homogenen Interaktionskonzept über mehrere Werkzeuge hinweg spielen Zoomtechniken eine tragende Rolle. Wie bereits einleitend ausgeführt sehen wir in dem Einsatz von Zoomtechniken einen entscheidenden Vorteil gegenüber klassischen Interaktions- und Navigationskonzepten. Die zu Grunde liegende Annahme wurde von Perlin und Fox geäußert: "navigation in information spaces is best supported by tapping into our natural spatial and geographic ways of thinking" (Perlin \& Fox 1993). Der Einsatz von semantischem Zooming nach (Perlin \& Fox 1993), bei welchem der durch die Vergrößerung zur Verfügung stehende Platz durch eine Anreicherung mit Information sinnvoll verwendet wird, bietet darüberhinaus den Vorteil, dass der Benutzer durch die Zoominteraktion implizit den Grad seines Interesses ausdrücken kann. Mittlerweile finden Zoomtechniken in immer mehr Anwendungen Verwendung, beispielsweise in Kartenapplikationen wie Google Earth oder auch im Webbrowser Safari des iPhones.

Hinsichtlich der Interaktion mit Punktdiagrammen konnte bereits im Filmfinder durch die Manipulation von Schiebereglern eine Skalierungsänderung durchgeführt werden. Diese Interaktion ist jedoch eher umständlich, da indirekt und getrennt nach X- und Y-Achse. In der Arbeit von (Buering et al. 2006) zeigte sich zudem das Potential von FischaugeVerzerrungstechniken. Bei diesen kann der Benutzer zunächst einen Bereich einschränken, welcher daraufhin vergrößert wird. Gleichzeitig bleibt der Kontext erhalten, in dem die außerhalb des Bereichs liegenden Punkte zusammengedrängt im Randbereich angezeigt werden. In einem Experiment wurde die Technik insbesondere aufgrund der Möglichkeit, sich schnell auf einen interessanten Bereich zu fokussieren von den Benutzern klar gegenüber 
einem rein geometrisch-semantischem Zoom bevorzugt. Entsprechend diesen empirischen Erkenntnissen wurde die Zoominteraktion im HyperScatter gestaltet.

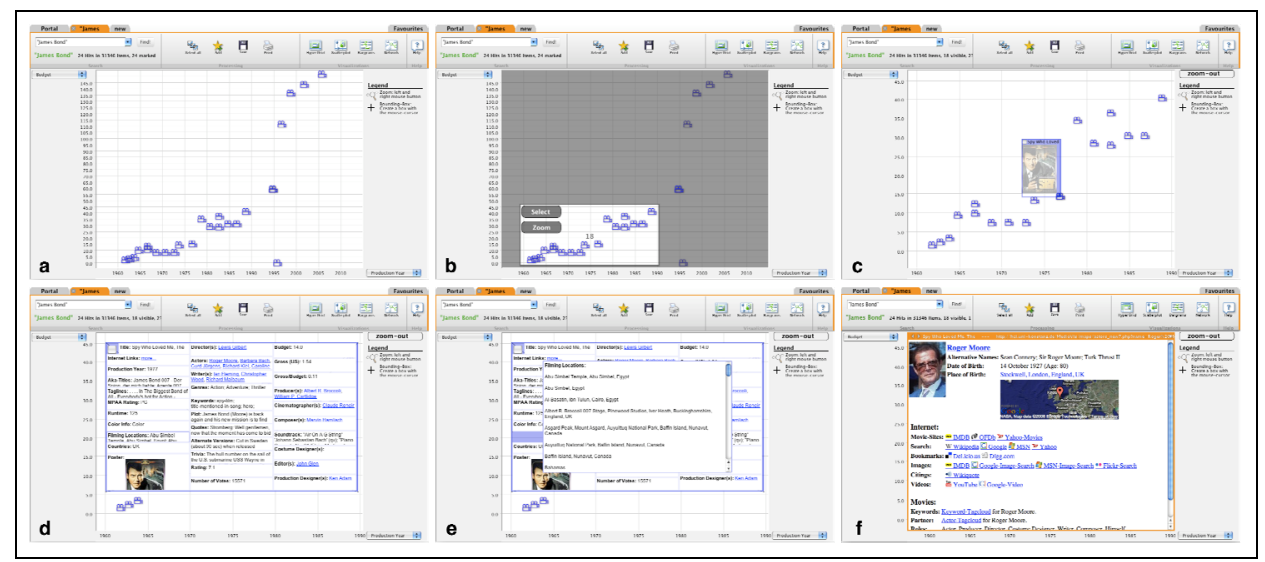

Abbildung 1: a) Übersicht HyperScatter, b) Bounding Box, c) MouseOver Detailinformationen, d) halbtransparentes Detailfenster für Datenpunkte, e) Attributfenster groß gezoomt, f) Internetbrowser Integration

Der Benutzer kann durch Gedrückt halten der Maus ein Rechteck um interessante Datenpunkte ziehen (Bounding-Box Zoom, Abbildung 1b). Daraufhin erscheinen direkt an dieses Rechteck angedockt zwei Schaltflächen, welche dem Benutzer die Wahl zwischen einer Zoom-Operation oder einer Selektionsoperation ermöglichen. Letztere ermöglicht die Auswahl und das Hinzufügen von Datenpunkten zu persönlichen Favoriten, welche über mehrere Suchen hinweg gesammelt werden können. Mittels einer Schaltfläche am rechten Rand des HyperScatters können die Zoomoperationen zudem wieder rückgängig gemacht werden. Der Zugriff auf einen Datenpunkt erfolgt entsprechend durch das Anklicken dieses mit der linken Maustaste, woraufhin ein halbtransparentes Fenster animiert aufgezoomt wird (Abbildung 1d). Entsprechend der Interaktion in der HyperGrid erfolgt ein rauszoomen durch Drücken der rechten Maustaste. Um allerdings ein unnötiges Rein- und Rauszoomen zu unterbinden, wird direkt beim Überfahren eines Datenpunktes mit der Maus bereits ein kleines Fenster aufgezoomt, welches das Filmposter sowie den Titel des Films zeigt (Abbildung 1c). Weiterhin ist eine Selektion des Objekts durch Anklicken einer Checkbox möglich. Das Fenster verschwindet automatisch wieder, sobald die Maus weiterbewegt wird. Hierdurch ist es dem Benutzer möglich, schnell und ohne explizite Interaktion den Datenraum zu durchstöbern.

\subsection{Darstellung und Exploration von Datenpunkten - Attributraum und Browserintegration}

Punktdiagramme eignen sich zwar hervorragend für die Analyse eines Informationsraums, jedoch können sie neben den beiden Achsendimensionen und eventuellen Farb- oder Symbolkodierungen zunächst keine weiteren Metadaten darstellen. Im Filmfinder wurde dies über Pop-Up Fenster gelöst, in den vorgestellten Arbeiten von Buering et al. entweder über 
einen geometrisch-semantischen Zoom oder wie auch im HyperScatter über animiert großzoomende Fenster. Bei jeder dieser Lösungen bleibt jedoch die Frage offen, wie daraufhin die Metadaten angeordnet und dem Benutzer präsentiert werden. Um diesem Problem zu begegnen greift der HyperScatter auf die Attributraummodellierung zurück. Diese ermöglicht ein einfaches Clustern der Metadaten hinsichtlich ihrer semantischen Nähe zueinander und wird in der HyperGrid dazu verwendet, die Spalten und deren Inhalte zu definieren einfach gesagt: welches Attribut erscheint wo und wann. Um diese Modellierung auf den HyperScatter zu übertragen, werden diese Cluster in dem aufgezoomten Detailfenster dem Benutzer in einer spaltenähnlichen Darstellung präsentiert - hierdurch wird die visuelle Metapher einer Tabelle aus dem HyperGrid Konzept wieder aufgegriffen und eine nahtlose Integration in das MedioVis Gesamtkonzept gewährleistet (siehe Abbildung 2).

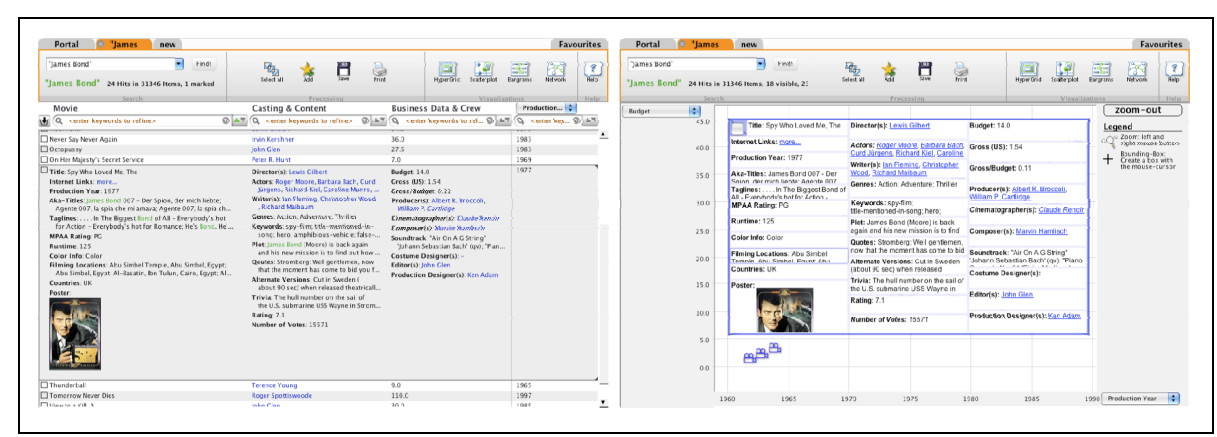

Abbildung 2: links HyperGrid und rechts HyperScatter

Ein generelles Problem von den im Filmfinder verwendeten Pop-Up Fenstern ist die schlechte Skalierung mit steigender Anzahl von Metadaten. Hier setzt der HyperScatter das Konzept einer zoombaren Benutzerschnittstelle konsequent fort.Je nach Anzahl der Attribute werden zunächst nur jeweils wenige Informationen zu den Metadaten angezeigt - der Benutzer hat aber die Möglichkeit, entsprechend seinem persönlichen Interesse, in einzelne Attribute per Mausklick weiter hinein zu zoomen (siehe Abbildung 1e).Dabei wird wiederum ein Fenster animiert aufgezoomt, in welchem das Attribut vollständig dargestellt werden kann. Entsprechend dem Gesamtinteraktionskonzept erfolgt ein Herauszoomen aus diesem Fenster wiederum per Rechtsklick. Basierend auf dem HyperGrid Konzept lassen sich zudem nahezu beliebige Hyperlinks auf eigene oder externe Web-Services und Seiten in einem derartigen Detailfenster darstellen. Durch diese Browserintegration können beispielsweise weiterführende Informationen zu Schauspielern ebenso angezeigt werden wie ein Link auf eine Youtube-Suche nach entsprechenden Filmtrailern (siehe Abbildung 1f).

\section{Evaluation: HyperScatter vs. HyperGrid}

Um das vorgestellte Konzept auf seine Gebrauchstauglichkeit hin zu prüfen, wurde eine Evaluationsstudie durchgeführt. Neben dem Ziel, Probleme in der Benutzbarkeit zu finden 
sollte vor allen Dingen auch untersucht werden, inwieweit der HyperScatter als alleinstehendes Werkzeug tragfähig ist - sprich ob er in einem MedioVis Gesamtsystem eher alternativ oder gemeinsam mit Visualisierungen wie der HyperGrid angeboten werden sollte und wo die aufgabenspezifischen Stärken und Schwächen liegen. Als Benchmarksystem wurde eben jene HyperGrid verwendet, die bereits in Evaluationsstudien zeigen konnte (Jetter et al. 2005), dass sie diese Voraussetzungen erfüllt. Entgegen der klassischen Vorgehensweise wurde jedoch kein Laborexperiment durchgeführt, sondern eine Längsschnittstudie im Feld über den Zeitraum von zwei Wochen. Hierdurch war es möglich, die Systeme im Echteinsatz zu beobachten und einen Beitrag dahingehend zu leisten, die Methodik von Längsschnittstudien weiter $\mathrm{zu}$ verfeinern. Angelehnt ist das Design hierbei an das von (Shneiderman\&Plaisant 2006) vorgeschlagene Modell für Langzeit-Usability Feldstudien, welches anhand einer Case Study auch eine mögliche Vorgehensweise illustriert. Demzufolge ist ein wichtiger Aspekt die Kombination unterschiedlicher Methoden zur Datenerhebung. Im vorliegenden Fall wurde auf Interviews und Tagebuchtechniken sowie auf die Erhebung von Interaktionsdaten durch Logfiles zurückgegriffen.

\subsection{Design}

Acht Versuchspersonen, Studenten an der Universität Konstanz, wurden für die zweiwöchige Studie ausgewählt. Alle wiesen ein hohes Filminteresse auf, was aufgrund des gewählten Informationsraumes „Kinofilme“ ein wichtiger Aspekt war. In der ersten Interviewsession wurde ihnen eine Kopie des Systems ausgehändigt mit der Aufforderung, dieses anstelle ihrer bisherigen Vorgehensweise zur Filmrecherche einzusetzen. Als Entschädigung erhielten die Versuchspersonen 20€. In einigen entscheidenden Punkten weicht das gewählte Design von den in der Literatur bekannten Vorgehensweisen ab. Es erfolgte ein Systemwechsel nach zwei Wochen inklusive Counterbalancing. Zur Steigerung der Motivation der Benutzung wurden einfache Wochenaufgaben vergeben, die auch zur Kontrolle, ob wichtige Funktionen gefunden und genutzt wurden, dienten. Für eine Analyse der Bearbeitungsdauer eigneten sie sich jedoch aufgrund des Umfangs und der Gestaltung nicht. Die Ausgabe erfolgte erst nach dreitägiger freier Benutzung. Es bestand die Möglichkeit, in Woche zwei mit beiden Systemen zu arbeiten, was zudem eine implizite Präferenzäußerung ermöglichte. Die Tagebuch-Protokolle bestanden aus knapp zwei Seiten, wobei hier gefragt wurde, für welche Art von Aufgabe das System verwendet wurde (beispielsweise explorative Suche oder Suche nach bestimmten Film), welche Probleme aufgetreten waren, inwieweit externe Inhalte verwendet wurden und ob die Benutzung Spaß gemacht hatte. Das Dokument sollte direkt nach jeder Benutzung ausgefüllt werden. Dies ermöglichte eine Mikro-Perspektive auf die Systemnutzung. Es erfolgten Interviews zu Beginn, bei Systemwechsel und am Ende der Studie. Ziel hierbei: Systeme einführen, Demonstration von Problemen am Objekt. Darüberhinaus eine Validierung von Benutzungsproblemen die im Tagebuch vermerkt worden waren sowie die Erhebung einer vom Benutzer reflektierten Makro-Perspektive auf die Systemnutzung. Ziel dieses Designs war es, zum einen die freie Benutzung der Systeme zu fördern und hierdurch Erkenntnisse zu gewinnen, die so in einem Labortest nicht möglich sind. Zum anderen sollten durch die abwechselnde Benutzung beider Werkzeuge die Versuchspersonen in die Lage versetzt werden, eine abschließende Bewertung vorzunehmen und jeweilige Vor- und 
Nachteile abzuwiegen. Die Kombination der Methoden ermöglicht es zudem, die jeweiligen, zumeist qualitativen Erkenntnisse mehrfach zu validieren.

\section{$3.2 \quad$ Testsysteme}

Als Informationsraum wurde ein Abzug der Datenbank der Internet Movie Database (www.IMDb.com) verwendet. Weiterhin wurden zu Schauspielern und Filmen weitere Links zu Webservices angeboten, beispielsweise zu Youtube, um nach Trailern zu suchen oder auch zur IMDb direkt. Eine Textfeldsuche ermöglichte bei beiden Systemen die Eingrenzung des Informationsraumes. Die HyperGrid wurde in der aktuellen Version verwendet, wie sie in Abbildung 2 zu sehen ist. Der Benutzer erhält die Ergebnisse in einer tabellenbasierten Ansicht in Zeilen angeordnet - die Metadaten sind entsprechend dem Attributraummodell in drei Spalten angeordnet. Durch Linksklick in einzelne Zellen können diese vergrößert werden und weitere Metadaten werden sichtbar. Detailinformationen zu den Metadaten können in zellüberlagernden Fenstern dargestellt werden, welche durch Klick auf den Attributnamen erscheinen. Darüberhinaus ermöglichen Links zu Schauspielern oder dem Film selbst die Anzeige eines Internetbrowserfensters innerhalb der Zelle, welches zudem bis zum Vollbild vergrößert werden kann. Mittels eines Tabellenfilter Konzepts ist zudem ein nachträgliches Einschränken der Datenmenge möglich - hierzu werden Begriffe in die Felder unter den Spaltenbeschriftungen eingetragen, woraufhin die Ergebnisse entsprechend gefiltert werden. Die vierte Spalte ermöglicht es dem Benutzer zudem, ein beliebiges Attribut für Vergleichsoperationen an die Oberfläche zu holen. Im HyperScatter kann der Benutzer neben den bereits vorgestellten Funktionen die Belegung der Achsen im Koordinatensystem ändern.

\subsection{Ergebnisse}

Neben dem Vergleich der beiden Systeme werden insbesondere noch die Benutzungsprobleme beim HyperScatter beleuchtet sowie auf Vor- und Nachteile der Methode eingegangen. Insgesamt haben von den acht Versuchspersonen fünf Teilnehmer die Studie vollständig absolviert. Eine Person ist bereits zu Beginn ausgestiegen, zwei weitere nach einer Woche, wobei diese das System jeweils kaum verwendet hatten.

Die durchschnittliche Sessiondauer lag bei Benutzung des HGs mit 15,67 Minuten höher als beim HS (9,1 Minuten, Analyse über Sessions statistisch signifikant $F(1,74)=5,362$, $\mathrm{p}=0,023)$. Aufgrund der höheren Anzahl an Sessions mit dem HS unterschied sich die Benutzung in der Gesamtdauer jedoch nur unwesentlich mit 454 Minuten (HG) zu 428 Minuten (HS). Dies deutet in beiden Fällen darauf hin, dass die Systeme durchaus auch für komplexere Suchen verwendet wurden. Gerade die Möglichkeit des Stöberns wurde zudem von den Probanden als Qualitätskriterium des HyperScatters genannt, wohingegen die HyperGrid eher für die gezielte Suche nach einem Titel Vorteile böte. Die Integration von externen Webservices erwies sich ebenfalls als sinnvoll: in 27 Protokollen (17 von 39 bei der HG, 10 von 24 beim HS) wurde von den Teilnehmern angegeben, eingebundene externe Quellen verwendet zu haben, was sich durch die Analyse der Logfiles auch bestätigen lies. Weitere, nicht angebotene Quellen musste nur in sechs Fällen zu Rate gezogen werden. In den Tage- 
büchern und Interviews wurde dies von den Benutzern zudem als ein positiver Aspekt der Systeme hervorgehoben - sowohl hinsichtlich der Auswahl als auch der Form der Integration der Quellen. Auf einer 5-Punkt-Skala konnte in den Tagebüchern zudem angekreuzt werden, $\mathrm{ob}$ und wie viel $\mathrm{Spa} ß$ die Benutzung bereitete. Hierbei ergab sich ein leichter, jedoch nicht signifikanter Vorteil zu Gunsten des HyperScatter von 3,72 zu 3,56. Bei der abschließenden Frage nach der Präferenz zeigte sich ebenfalls ein ausgeglichenes Bild mit einem 3:2 Votum zu Gunsten der HyperGrid. Als Gründe für die Bevorzugung der HyperGrid wurde genannt, dass hiermit eine bessere Suche nach einem spezifischen Film oder Schauspieler möglich ist, da die Tabelle einen schnelleren Einblick in die Daten liefert. Im HyperScatter sind zunächst lediglich die Metadaten mit denen die Koordinatenachsen belegt wurden, sichtbar - nicht jedoch Attribute wie der Titel des Films. Als Gründe die für den HyperScatter sprechen wurden genannt, dass dieser komplexere Aufgaben besser unterstützt sowie für den Vergleich von Daten besser geeignet ist. Zudem sei er ästhetisch ansprechender gestaltet. Insgesamt wurde das Zooming Konzept in beiden Darstellungen sehr begrüßt - die Interaktion bereitete keinem der Teilnehmer Probleme und auch der Wechsel zwischen den Systemen verlief reibungslos - wobei hier insbesondere das einheitliche Interaktionsdesign das Erlernen des zweiten Systems begünstigte. Ebenso wurde die übersichtliche Darstellung gelobt eine Versuchsperson nannte dies als expliziten Vorteil gegenüber der IMDb, bei welcher man „rumscrollen“ und „rumklicken“ müsse. Sowohl in HG als auch HS wurde die Möglichkeit der freien Belegbarkeit einer Spalte bzw. der Achsen hervorgehoben. Dies lässt sich wiederum auch an den Logfiles sehen, wurde doch die Änderung der Achsenbelegung in 25 der 47 Sessions angewendet. Interessant war auch die mehrfache Äußerung, dass doch eine Kombination aus beiden Darstellungen sinnvoll wäre, ohne dass auf das Gesamtsystem MedioVis hingewiesen worden wäre. Sämtliche Teilnehmer konnten sich vorstellen, die Werkzeuge auch auf Dauer einzusetzen - zwei von Ihnen setzten dieses Vorhaben auch in die Tat um und haben mittlerweile in weiteren fünf Sessions mit den Systemen gearbeitet.

\section{Usability Probleme des HyperScatter im Detail}

Die Studie hat neben diesen positiven Aspekten aber auch eine Reihe von Benutzungsproblemen offenbart. Es wurden insgesamt 21 Probleme bei der HyperGrid festgestellt und 18 bei dem HyperScatter - allerdings unterscheiden sich diese sehr stark in ihrer Granularität, so wurden von den Teilnehmern sowohl kleine Detailprobleme als auch konzeptbedingte Probleme festgestellt. Als schwerwiegendste Probleme des HS wurden identifiziert, dass dieser bei vielen Treffern zur Unübersichtlichkeit neigt und eine Interaktion mit den Datenpunkten zwingend notwendig ist, um weitere Informationen zu erhalten. Daher sei er auch schlecht geeignet, um genau einen Film zu finden. Weiterhin wurde die fehlende Verknüpfung zwischen Suchbegriff und Treffern kritisiert. In vielen Fällen war den Benutzern nicht klar, wieso manche Treffer überhaupt angezeigt wurden, da keine Verknüpfung mit dem Suchbegriff ersichtlich war. Dies trat insbesondere bei der Suche nach Personen auf, da dann auch Filme gefunden wurden, bei denen diese lediglich einen Gastauftritt hatten und somit auch nicht prominent in den Daten auftauchen.

\section{Zeitliche Aspekte \& Methodenkritik}

Insgesamt muss an dieser Stelle gesagt werden, dass die Dauer von zwei Wochen letztlich zu gering war, um Tendenzen in der Benutzung zu erkennen - beispielsweise ob komplexere 
Funktionen erst nach einer gewissen Zeit verstärkt eingesetzt werden. Interessant ist aber, dass in der ersten Session die Versuchspersonen in den Protokollen lediglich fünf Benutzungsprobleme entdeckten - eine längere Nutzungsdauer die Erkennungsrate also deutlich steigern konnte. Von der Möglichkeit, das erste System auch in der zweiten Woche zu nutzen machte leider lediglich eine Versuchsperson Gebrauch - wodurch sich hier keine Aussagen hinsichtlich einer implizit geäußerten Präferenz machen lassen. Die Wochenaufgaben scheinen zudem die Benutzung positiv gesteigert zu haben. Aus den Benutzungsprotokollen geht beispielsweise hervor, dass einige Funktionen bei der HyperGrid (Sortierung und Tabellenfilter) erst durch diese entdeckt wurden. Bezüglich der Methode insgesamt ist zu sagen, dass die Kombination aus den drei Einzelmethoden deutliche Vorteile brachte. So konnten ebenso unterschiedliche Probleme identifiziert werden wie eine mehrfache Validierung dieser. Auch bei den Versuchspersonen kam die Studie gut an, sahen sie sich doch viel mehr in der Rolle des Testers und hatten Freude daran, Feedback zu geben. Ein weiterer Aspekt, der bei der Durchführung beachtet werden sollte, ist dass die Benutzer nicht zwischen Daten und Benutzeroberflächen Problemen unterscheiden. So führten einige kleinere Fehler zu frustrierten Tagebuchprotokollen und viele der genannten Probleme ließen sich auf die Daten beziehungsweise deren Aufbereitung zurückführen - beispielsweise wurde das Fehlen der Rolleninformationen bei den Schauspielerdaten mehrfach bemängelt. Gegenüber einem klassischen Usability Test ist zudem auch der Aufwand deutlich gesteigert - auf der anderen Seite lassen sich jedoch Probleme identifizieren, die im Labor aufgrund der künstlichen Situation kaum zu entdecken sind und zum anderen lassen sich validere Aussagen über die Praxistauglichkeit treffen.

\section{$4 \quad$ Diskussion und Ausblick}

Abschließend lässt sich festhalten, dass sowohl der HyperScatter als auch die HyperGrid in der Feldstudie überzeugen konnten und die Teilnehmer von den Möglichkeiten durchaus beeindruckt waren. Allerdings hat sich auch gezeigt, dass der HyperScatter als alleinstehendes Werkzeug in seiner jetzigen Form noch nicht ausreichend ist, da er Aufgaben wie die gezielte Suche nach einzelnen Objekten nur schlecht unterstützt. Hierbei könnten insbesondere erweiterte Filterkonzepte, wie beispielsweise die dynamic-queries von Shneiderman, Abhilfe schaffen. Darüberhinaus könnte die Zoominteraktion verbessert werden, indem der verwendete Bounding-Box Zoom mit einem geometrisch-semantischen Zoom kombiniert wird. Hierdurch könnten dem Benutzer bei ausreichendem Platz bereits erste Detailinformationen zu einzelnen Datenobjekten angezeigt werden - ein sequentielles Überfahren dieser, um herauszufinden, welches das Gesuchte ist, könnte dementsprechend entfallen. In weiteren Forschungsarbeiten soll daher das Potential eines derartig gestalteten Punktdiagramms weiter untersucht werden. Zudem soll analysiert werden, inwieweit das Zusammenspiel der beiden Darstellungsformen in der Praxis funktioniert, wenn diese in Kombination angeboten werden - gerade für ungeschulte Anwender muss hier schnell ersichtlich sein, für welche Art von Aufgabe welche Darstellung besser geeignet ist oder auch wie diese sich gegenseitig ergänzen können. Die verwendete Evaluationsmethodik hinterlässt einen positiven Eindruck, konnte sie doch Erkenntnisse liefern, die so im Labor nur schwer möglich gewesen wären - 
gerade hinsichtlich der Praxistauglichkeit der Systeme. Allerdings stellt sie sehr hohe Anforderungen an die Reifegrad und die Stabilität der Systeme - ansonsten besteht die Gefahr, dass die Teilnehmer zum einen frustriert werden und zum anderen lediglich technische Probleme melden. Zukünftig sollte die Dauer bei einer vergleichenden Studie auf vier Wochen erhöht werden, um auch untersuchen zu können, wie sich die Benutzung über die Zeit eventuell verändert. Ebenso scheint eine höhere Teilnehmerzahl unausweichlich, um Ausfälle besser kompensieren zu können. Auch eine Kombination mit einem klassischen Usability Test ist denkbar.

\section{$5 \quad$ Literaturverzeichnis}

Ahlberg, C. and Shneiderman, B. (1994). Visual information seeking using the FilmFinder. CHI '94: Conferencecompanion on Human factors in computing systems', ACM,New York, NY, USA.

Buering, T., Gerken, J. and Reiterer, H. (2006).User Interaction with Scatterplots on Small Screens - A Comparative Evaluation of Geometric-Semantic Zoom and Fisheye Distortion. IEEE Transactions on Visualization andComputer Graphics (12:5), pp. 829-836.

Dunlop, M. D., Davidson, N. (2000).Visual information seeking on palmtop devices. In Proceedings of HCI2000, volume 2, pages 19-20.

Furnas, G.W. (1986). Generalized fisheye views. In CHI '86:Proceedings of the SIGCHI conference on Human factors incomputing systems, pages 16-23, New York, NY, USA,ACM Press.

Grün, C., Gerken, J., Jetter, H., König, W. and Reiterer, H. (2005). MedioVis - a User-Centred Library Metadata Browser. Proceedings ECDL 2005, Springer Verlag.

Jetter, H., Gerken, J., König, W. A., Grün, C. and Reiterer, H. (2005). HyperGrid - Accessing Complex Information Spaces, HCI-UK’05, Springer Verlag.

Limbach, T., Reiterer, H., Klein, P. and Müller, F. (2003).VisMeB: A visual Metadata Browser.Interact 2003, IOSPress, pp. 993-996.

Meyer, J., Shinar, D. (1991). Perceiving Correlations from Scatterplots. Proceedings of the Human Factors Society 35th Annual Meeting: Visual Performance: Displaying Information, p1537-1540.

North, C., Shneiderman, B.(2000). Snap-together Visualization: Can Users Construct and Operate Coordinated Visualizations?.Int. J. Human-Computer Studies 53.

Perlin, K. and Fox, D. (1993). Pad: an alternative approach to the computer interface.SIGGRAPH '93, ACM, New York, NY, USA, pp. 57-64.

Reiterer, H., Jetter, H. C., König, W., Gerken, J., Grün, C. (2005).Zoomtechniken zur Exploration komplexer Informationsräume am Beispiel HyperGrid. Proceedings Mensch \& Computer 2005: Kunst und Wissenschaft - Grenzberschreitungen der interaktiven ART, LNCS, Oldenbourg-Verlag.

Shneiderman, B. and Plaisant, C. (2006). Strategies for evaluating information visualization tools: multi-dimensional in-depth long-term case studies. AVI 2006 Workshop: BELIV'06, ACM.

Waldeck, C., Balfanz, D (2004). Mobile liquid 2d scatter space (ml2dss). In IV'04: Proceedings of the Eighth International Conference on Information Visualisation, pages 494-498. IEEE. 